\title{
Optimization of Extraction Process and the Antioxidant Activity of Phenolics from Sanghuangporus baumii
}

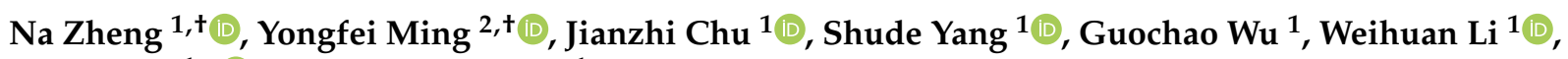 \\ Rui Zhang ${ }^{1, *}$ (D) and Xianhao Cheng ${ }^{1, *}$
}

1 Shandong Key Lab of Edible Mushroom Technology, School of Agriculture, Ludong University, Yantai 264025, China; zn1221914@163.com (N.Z.); chu_jianzhi@163.com (J.C.); sdyang68@126.com (S.Y.); wuguochaoo@163.com (G.W.); lwh1979@163.com (W.L.)

2 School of Life Science, Ludong University, Yantai 264025, China; mingyf@163.com

* Correspondence: zhlrui@163.com or 2755@ldu.edu.cn (R.Z.); chengxianhao@sohu.com (X.C.); Tel.: +86-0535-6664665 (R.Z. \& X.C.)

+ These authors contributed equally to this work.

check for

updates

Citation: Zheng, N.; Ming, Y.; Chu, J.; Yang, S.; Wu, G.; Li, W.; Zhang, R.; Cheng, X. Optimization of Extraction Process and the Antioxidant Activity of Phenolics from Sanghuangporus baumii. Molecules 2021, 26, 3850. https://doi.org/10.3390/ molecules 26133850

Academic Editors: Liangcai Peng, Feng Peng and Wanbin Zhu

Received: 21 May 2021

Accepted: 22 June 2021

Published: 24 June 2021

Publisher's Note: MDPI stays neutral with regard to jurisdictional claims in published maps and institutional affiliations.

Copyright: (C) 2021 by the authors. Licensee MDPI, Basel, Switzerland. This article is an open access article distributed under the terms and conditions of the Creative Commons Attribution (CC BY) license (https:/ / creativecommons.org/licenses/by/ $4.0 /)$.

\begin{abstract}
Sanghuangporus baumii, is a widely used medicinal fungus. The polyphenols extracted from this fungus exert antioxidant, anti-inflammatory, and hypoglycemic effects. In this study, polyphenols from the fruiting bodies of $S$. baumii were obtained using the deep eutectic solvent (DES) extraction method. The factors affecting the extraction yield were investigated at different conditions. Based on the results from single-factor experiments, response surface methodology was used to optimize the extraction conditions. The scavenging ability of the polyphenols on $\bullet \mathrm{OH}, \mathrm{DPPH}$, and $\mathrm{ABTS}^{+}$was determined. The results showed that the DES system composed of choline chloride and malic acid had the best extraction yield $(6.37 \mathrm{mg} / \mathrm{g})$. The optimal extraction parameters for response surface methodology were as follows: $42 \mathrm{~min}, 58{ }^{\circ} \mathrm{C}, 1: 34$ solid-liquid $(\mathrm{mg} / \mathrm{mL})$, and water content of $39 \%$. Under these conditions, the yield of polyphenols was the highest $(12.58 \mathrm{mg} / \mathrm{g})$. At $0.30 \mathrm{mg} / \mathrm{mL}$, the scavenging ability of the polyphenols on $\bullet \mathrm{OH}, \mathrm{DPPH}$, and $\mathrm{ABTS}^{+}$was $95.71 \%, 91.08 \%$, and $85.52 \%$, respectively. Thus, the method using DES was more effective than the conventional method of extracting phenolic compounds from the fruiting bodies of S. baumii. Moreover, the extracted polyphenols exhibited potent antioxidant activity.
\end{abstract}

Keywords: polyphenols; Sanghuangporus baumii; deep eutectic solvent; response surface methodology; antioxidant

\section{Introduction}

Sanghuang is a popular medicinal fungus available in China, with a reputation of being "forest gold." Sanghuang has been used as traditional Chinese medicine for more than 2000 years and is recorded in many ancient Chinese books [1]. According to the Shennong Materia Medica Classic and Compendium of Materia Medica, its main effects are as following: relieving diarrhea, breaking and leaking down the belt, and relieving diarrhea by spleen deficiency [2]. Although ancient Chinese books indicate the use of Sanghuang as medicine, the species of Sanghuang that can be used cannot be identified solely based on their description in the literature, because of the existence of several varieties of Sanghuang. In recent years, scholars still have differences in opinion regarding the classification of Sanghuang. However, studies show that almost all varieties of Sanghuang exert several pharmacological effects.

In 2012, a new species was identified and named Inonotus Sanghuang Sheng H. Wu, T. Hatt. \& Y.C. Dai [3]. In 2016, Wu Shenghua et al. combined molecular biological identification and morphological analysis, and the genus Sanghuangporus Sheng H. Wu, L.W. Zhou \& Y.C. Dai were established. Currently, there are 14 species in this genus, of which nine are found in China [4]. Additionally, through the textual research of Chinese 
herbology studies, Bao Haiying et al. believe that Inonotus hispidus (Bull.) P. Karst. is the closest to Sanghuang. At the same time, Phelinus Quel., Enchir. Fung., and Inonotus P. Karst, which are close to the genus Sanghuangporus, can also be called Sanghuang [5]. To date, researchers continue to actively explore the classification and uses of Sanghuang. Sanghuangporus Baumii (Pilat) L.W. Zhou \& Y.C. Dai is a fungus belonging to the genus Sanghuangporus. Compared with S. Sanghuang Sheng H. Wu, T. Hatt. \& Y.C. Dai, S. baumii is known for its ability to grow easily; it has a short growth cycle and large-scale cultivation can be achieved.

Sanghuang contains several pharmacologically active compounds, including polysaccharides, flavonoids, pyrone, polyphenols, terpenoids, and sterols, which have been reported to cure diseases [6-9]. To date, the main polyphenols reported in Sanghuang include phellibaumin A, phelligridin C, phelligridin D, hypholomin B, protocatechualdehyde, interfungin $\mathrm{B}$, and chlorogenic acid [10-14]. Pharmacological studies have shown that polyphenols are associated with improving immunity and exert antitumor, antioxidant, antihyperglycemic, and hepatoprotective effects [15-18]. However, in recent years, researches on Sanghuang is mainly focused on polysaccharides and flavonoids, and very few studies have studied the polyphenols present in this fungus. Therefore, in-depth research on the extraction and pharmacological activity of the polyphenols of Sanghuang is well warranted.

Ethanol, methanol, and petroleum ether are some of the organic solvents commonly used for the extraction and separation of polyphenols from natural sources [19]. Other important extraction methods include ultrasound-assisted ethanol extraction [20], microwaveassisted methanol extraction [21], enzyme-assisted extraction [22], supercritical extraction [23], and subcritical water extraction [24]. These traditional methods have some disadvantages, such as extended extraction time, low purity of the extracted components, low extraction yield, and the possibility of environmental pollution. Therefore, it is necessary to identify new and green extraction solvents and design simple extraction schemes with high efficiency to replace some of the commonly used extraction solvents.

Deep eutectic solvents (DES) are green extraction solvents that can replace organic solvents. This system comprises a transparent solvent that is obtained by mixing two or more substances in a certain proportion. Abbott et al. [25] found that amides and quaternary ammonium salts mixed in a certain molar ratio can form low-melting eutectic mixtures having special solvent properties and named them DES. DES can be used for simple synthetic processes; moreover, they are inexpensive, non-toxic, low volatility, high solubility, nonflammability, environmentally friendly, reusable, and degradable [26], all of which conform to the principles and concepts of green chemistry. Owing to these unique properties, DES has attracted increasing attention from scholars and has been widely used in catalysis, organic synthesis, and analytical chemistry [27-29]. In this study, we selected a suitable eutectic solvent from among many DES to extract polyphenols from S. baumii. We optimized the extraction using a single-factor test combined with the response surface curve method. In addition, we performed in vitro experiments to determine the antioxidant activities of the extracted polyphenols. The findings of our study provide the corresponding theoretical basis for further research, including the development and utilization of Sanghuang in health products.

\section{Results}

\subsection{Effect of Different Eutectic Solvents on the Extraction of S. baumii Polyphenols}

The extraction yields of polyphenols varied to some extent because the hydrogen donor for each combination of DES differed. To determine the optimal composition of DES to extract $S$. baumii polyphenols, a total of 8 DES combinations were selected in this experiment. At the same time, different concentrations of ethanol were selected as controls to more directly reflect the differences between the two extraction systems. First, using a solid-liquid ratio of $50 \mathrm{mg} / \mathrm{mL}$, ultrasonic-assisted extraction time of $30 \mathrm{~min}$, a temperature of $50{ }^{\circ} \mathrm{C}$, and an extraction power of $200 \mathrm{~W}, 40 \mathrm{kHz}$, the effects of $50 \%, 60 \%, 70 \%, 80 \%$ 
and $90 \%$ ethanol on the yields of S. baumii polyphenols were compared. The experimental results are shown in Figure 1. We found that the highest yield of phenol was $4.20 \mathrm{mg} / \mathrm{g}$ when $60 \%$ ethanol was used as the extraction solvent.

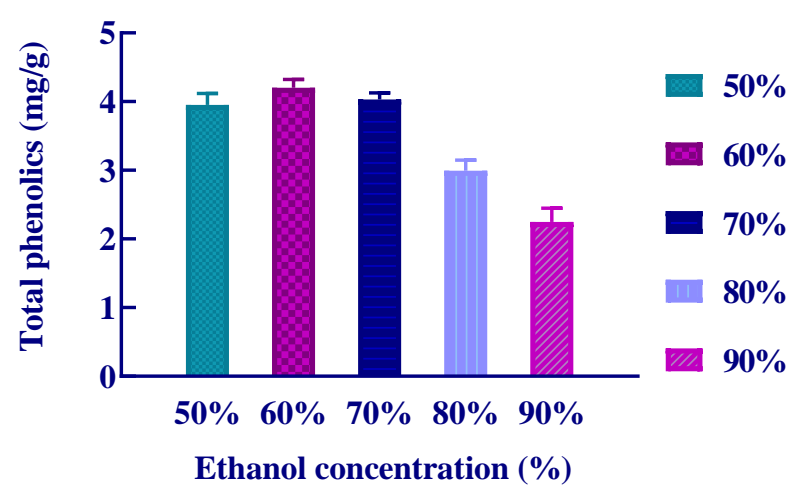

Figure 1. Effect of ethanol concentration on the extraction yield of S. baumii polyphenols.

Eight different DES with $40 \%$ water content were used to extract S. baumii polyphenols using a solid-liquid ratio of $50 \mathrm{mg} / \mathrm{mL}$, ultrasonic-assisted extraction for $30 \mathrm{~min}$, temperature of $50{ }^{\circ} \mathrm{C}$, and extraction power of $200 \mathrm{~W}, 40 \mathrm{kHz} ; 60 \%$ ethanol was used as a control. The results are shown in Figure 2. With the exception of the choline chloride and glycerol (DES-3) system, the extraction yield of other combinations of DES was better than that obtained using ethanol. Other combinations of DES led to yields higher than those obtained using ethanol extraction. The choline chloride and malic acid (DES-1) combination for the extraction of polyphenols led to the highest yield of $6.37 \mathrm{mg} / \mathrm{g}$. The extraction yield of choline chloride and malic acid DES was 1.5 times that of $60 \%$ ethanol. Therefore, DES can be used to replace organic solvents and show remarkable application potential in the extraction of polyphenols. Based on our findings, DES-1 was selected as the extraction solvent for subsequent experiments.

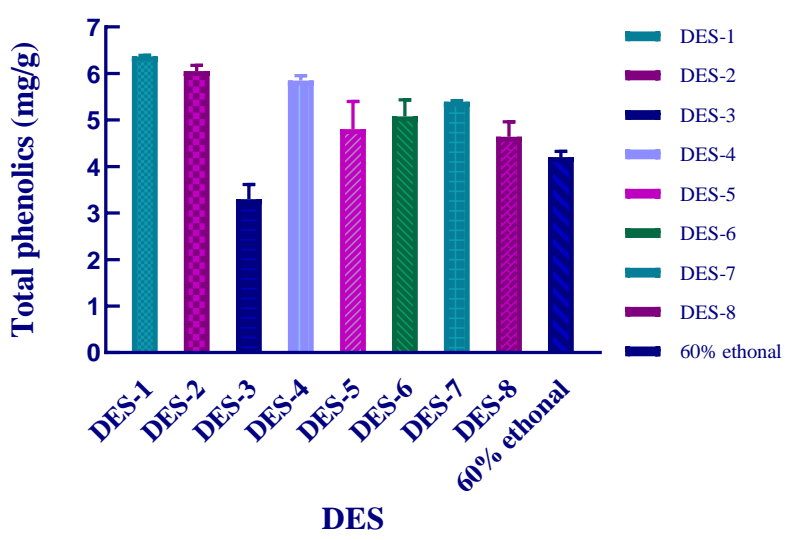

Figure 2. Effects of different DES on the extraction yield of polyphenols in S. baumii.

\subsection{Results from Single-Factor Experiments}

The results of the single factor test are shown in Figure 3. The experimental results show that when the water content was $40 \%$, the yield of the extracted polyphenols reached the maximum, shown in Figure 3A. When the extraction time was $40 \mathrm{~min}$, the polyphenols yield was not significantly different from that obtained using an extraction time of $50 \mathrm{~min}$. From the view of energy and cost savings, $40 \mathrm{~min}$ was selected as the optimal extraction time of $S$. baumii polyphenols, shown in Figure 3B. The polyphenols yield was the highest at $60^{\circ} \mathrm{C}$, shown in Figure $3 \mathrm{C}$. The polyphenols yield was the highest at a ratio of $40 \mathrm{mg} / \mathrm{mL}$, shown in Figure 3D. 


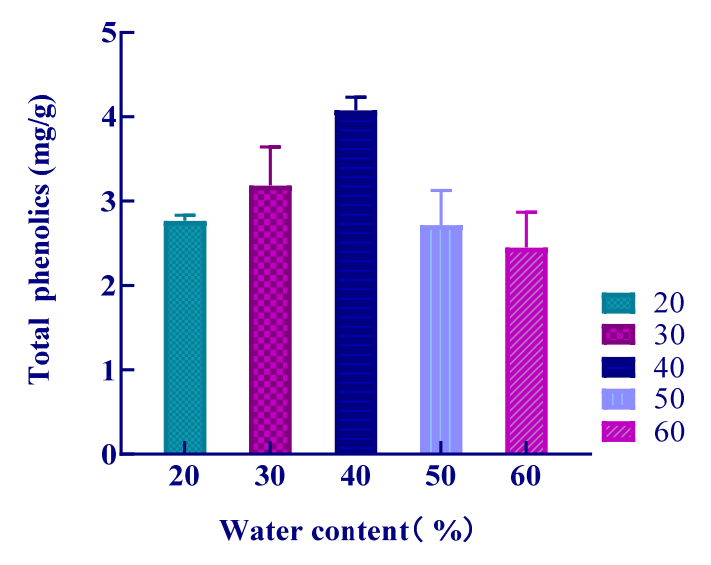

(A)

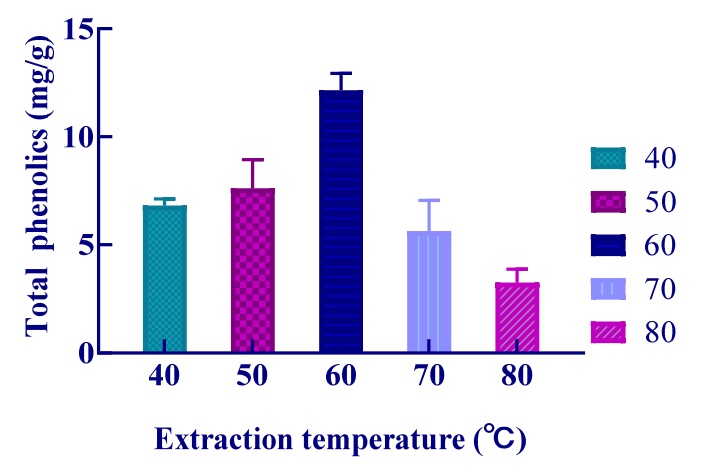

(C)

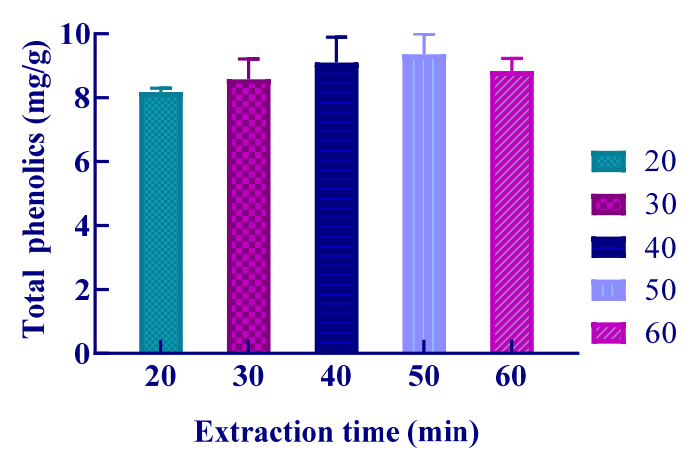

(B)

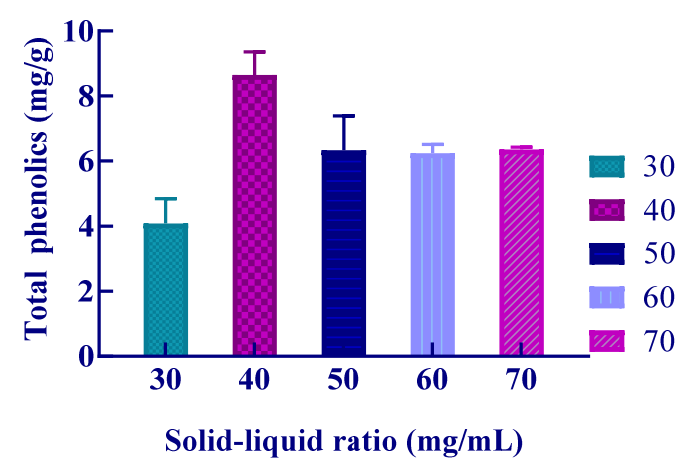

(D)

Figure 3. Effect of different extraction conditions on the extraction yield of polyphenols.

\subsection{Optimization of Polyphenols Extraction Using Response Surface Methodology}

The design plan and results obtained using Design-Expert 12 are shown in Table 1. Multiple linear regression analysis was conducted based on the results in Table 1, and the linear regression equation of the experiment was conducted as follows:

$$
\begin{aligned}
y= & -93.46+0.81 A+0.69 \mathrm{~B}+2.19 \mathrm{C}+0.72 \mathrm{D}+0.015 \mathrm{AB}-0.007 \mathrm{AC}-0.004 \mathrm{AD} \\
& -0.005 B D+0.002 C D-0.05 \mathrm{~A}^{2}-0.006 \mathrm{~B}^{2}-0.018 \mathrm{C}^{2}-0.006 D^{2}
\end{aligned}
$$

Results of the regression equation are shown in Table 2. From the equation, the correlation coefficient was determined to be $\mathrm{R}^{2}=0.9271$. For the regression model, $p<0.0001$ and reached an extremely significant level; the loss of fit term was $0.0716, p>0.05$. The loss of fit term is not significant, indicating that the selection of the model was reasonable and feasible, and could be used to analyze and predict the extraction of polyphenols. In the regression equation, $p$ values of $A$ and $C$ were $<0.05$, indicating that the two influencing factors of $A$ and $C$ had significant effects on the extraction yield of polyphenols. At the same time, through the comparison of the value of $F$, we can judge the influence of various factors on the extraction yield of $S$. baumii. The influence of each factor can be presented as follows: $\mathrm{C}>\mathrm{A}>\mathrm{B}>\mathrm{D}$. 
Table 1. Response surface experimental design scheme and results.

\begin{tabular}{|c|c|c|c|c|c|}
\hline Number & $\begin{array}{l}\text { Ratio of the Material } \\
\text { Liquid }(\mathrm{mg} / \mathrm{mL}) \mathrm{A}\end{array}$ & $\begin{array}{c}\text { Extraction } \\
\text { Time(min)B }\end{array}$ & $\begin{array}{c}\text { Extraction } \\
\text { Temperature }\left({ }^{\circ} \mathrm{C}\right) \mathrm{C}\end{array}$ & $\begin{array}{c}\text { The Water } \\
\text { Content(\%)D }\end{array}$ & $\begin{array}{l}\text { Polyphenols } \\
\text { Content }(\mathrm{mg} / \mathrm{g})\end{array}$ \\
\hline 1 & 40 & 40 & 70 & 30 & 8.39 \\
\hline 2 & 40 & 30 & 60 & 50 & 10.39 \\
\hline 3 & 40 & 40 & 60 & 40 & 12.37 \\
\hline 4 & 40 & 30 & 50 & 40 & 10.27 \\
\hline 5 & 30 & 40 & 60 & 50 & 11.43 \\
\hline 6 & 40 & 40 & 50 & 30 & 11.97 \\
\hline 7 & 40 & 50 & 60 & 30 & 12.09 \\
\hline 8 & 30 & 40 & 60 & 30 & 10.96 \\
\hline 9 & 50 & 40 & 60 & 30 & 10.77 \\
\hline 10 & 30 & 50 & 60 & 40 & 12.17 \\
\hline 11 & 40 & 30 & 70 & 40 & 8.27 \\
\hline 12 & 40 & 40 & 60 & 40 & 12.33 \\
\hline 13 & 30 & 40 & 70 & 40 & 10.74 \\
\hline 14 & 50 & 40 & 70 & 40 & 7.32 \\
\hline 15 & 40 & 40 & 60 & 40 & 11.92 \\
\hline 16 & 30 & 30 & 60 & 40 & 11.93 \\
\hline 17 & 40 & 40 & 50 & 50 & 11.12 \\
\hline 18 & 40 & 50 & 50 & 40 & 10.68 \\
\hline 19 & 40 & 50 & 60 & 50 & 10.51 \\
\hline 20 & 50 & 40 & 60 & 50 & 9.53 \\
\hline 21 & 40 & 40 & 60 & 40 & 11.73 \\
\hline 22 & 40 & 30 & 60 & 30 & 10.09 \\
\hline 23 & 50 & 50 & 60 & 40 & 10.96 \\
\hline 24 & 40 & 40 & 60 & 40 & 12.03 \\
\hline 25 & 50 & 30 & 60 & 40 & 10.14 \\
\hline 26 & 30 & 40 & 50 & 40 & 10.98 \\
\hline 27 & 40 & 50 & 70 & 40 & 8.45 \\
\hline 28 & 40 & 40 & 70 & 50 & 8.44 \\
\hline 29 & 50 & 40 & 50 & 40 & 10.22 \\
\hline
\end{tabular}

Table 2. Variance analysis of extraction yield using the regression model.

\begin{tabular}{cccccc}
\hline Sources Model & $\begin{array}{c}\text { Sum of Squares } \\
\mathbf{4 9 . 3 2 0}\end{array}$ & $\begin{array}{c}\text { DF } \\
\mathbf{1 4}\end{array}$ & $\begin{array}{c}\text { Mean Square } \\
\mathbf{3 . 5 2 0}\end{array}$ & $\begin{array}{c}\boldsymbol{F} \text {-Value } \\
\mathbf{1 2 . 7 2 0}\end{array}$ & $\begin{array}{c}\boldsymbol{p} \text {-Value } \\
<\mathbf{0 . 0 0 0 1}\end{array}$ \\
\hline A-A & 7.160 & 1 & 7.160 & 25.860 & 0.0002 \\
B-B & 1.180 & 1 & 1.180 & 4.280 & 0.0576 \\
C-C & 15.480 & 1 & 15.480 & 55.900 & $<0.0001$ \\
D-D & 0.687 & 1 & 0.677 & 2.440 & 0.1403 \\
AB & 0.084 & 1 & 0.084 & 0.304 & 0.5903 \\
AC & 1.770 & 1 & 1.770 & 6.390 & 0.0242 \\
AD & 0.731 & 1 & 0.731 & 2.640 & 0.1265 \\
BC & 0.013 & 1 & 0.013 & 0.048 & 0.8302 \\
BD & 0.884 & 1 & 0.884 & 3.190 & 0.0957 \\
CD & 0.202 & 1 & 0.203 & 0.731 & 0.4069 \\
A & 1.430 & 1 & 1.430 & 5.180 & 0.0392 \\
B & 2.490 & 1 & 2.490 & 9.010 & 0.0095 \\
C $^{2}$ & 20.040 & 1 & 20.040 & 72.350 & $<0.0001$ \\
D $^{2}$ & 2.760 & 1 & 2.760 & 9.970 & 0.0070 \\
Residual & 3.880 & 14 & 0.277 & & 0.0716 \\
Lack of fit & 3.580 & 10 & 0.358 & 4.820 & Not Significant \\
Pure Error & 0.297 & 4 & 0.074 & & \\
R $^{2}$ & 0.927 & & & & \\
\hline
\end{tabular}

A 3D response surface map was used to represent the interaction between various factors, shown in Figure 4. The interactions among various factors can be seen more 
intuitively from the 3D figure. The surfaces of factors $A$ and $C$ are relatively steep, while the changes in factors $B$ and $D$ are relatively gentle, indicating that $A$ and $C$ have a greater impact on the extraction yield of polyphenols.
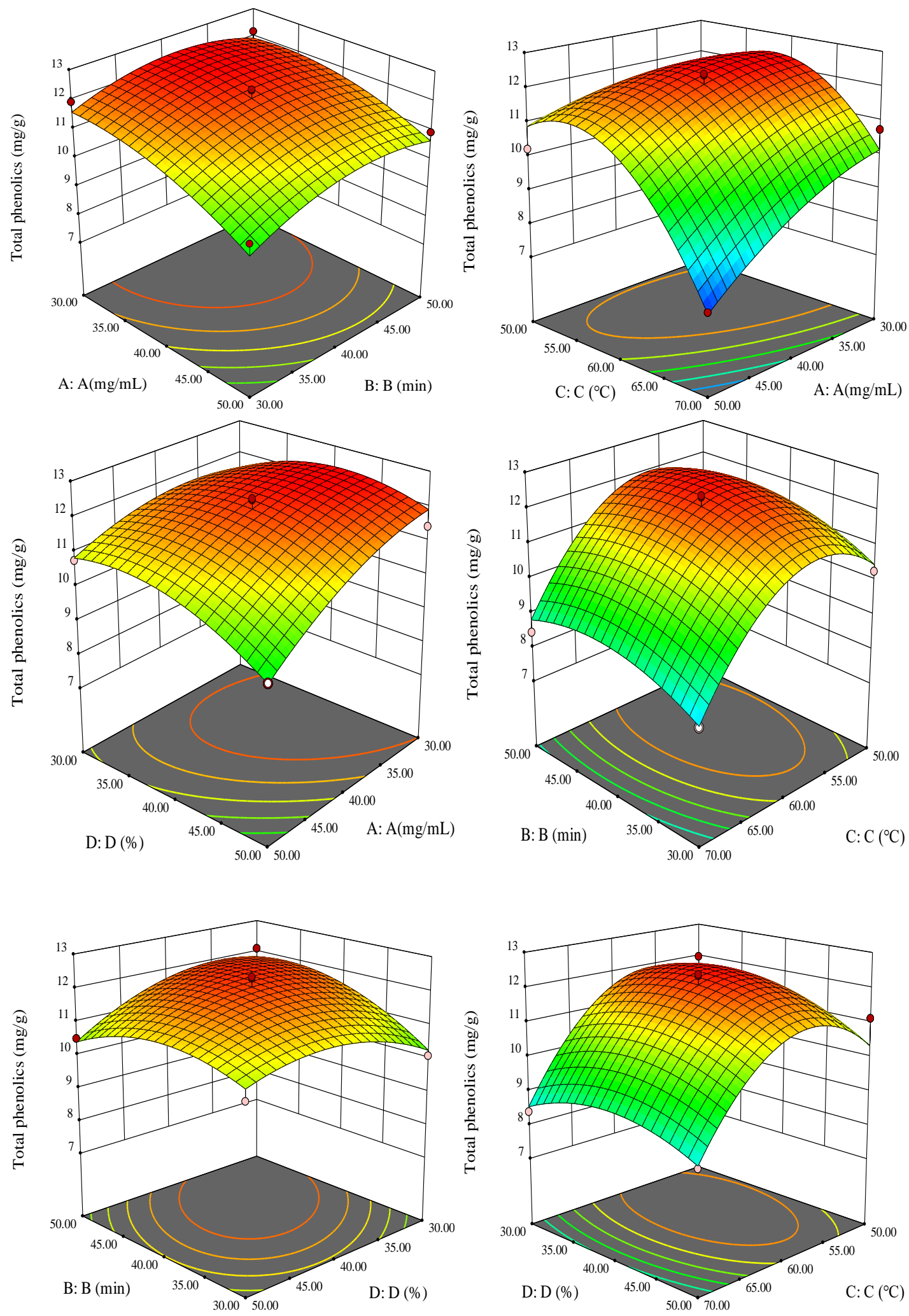

Figure 4. Response surface of the effects of the interaction of various factors on the extraction yield of S. baumii polyphenols. 
Four factors were analyzed using the Desk-Expert12, and the optimal extraction conditions were determined to be as follows: water content was $38.76 \%$, extraction time was $42.45 \mathrm{~min}$, extraction temperature was $57.72{ }^{\circ} \mathrm{C}$, and solid-liquid ratio was $34.35 \mathrm{mg} / \mathrm{mL}$. Using these optimal conditions, the predicted extraction yield of polyphenols was $12.48 \mathrm{mg} / \mathrm{g}$. To ensure the experimental feasibility, the optimal extraction conditions were modified as follows: water content was 39\%, extraction time was $42 \mathrm{~min}$, extraction temperature was $58^{\circ} \mathrm{C}$, and the solid-liquid ratio was $34 \mathrm{mg} / \mathrm{mL}$. Using these modified conditions, each experiment was performed in triplicate. The extraction yield of $S$. baumii polyphenols was $12.58 \mathrm{mg} / \mathrm{g}$ and the relative deviation was $0.85 \%$, both of which were consistent with the corresponding theoretical values. These findings indicated that our model was reasonable and feasible and could be used to predict and analyze the extraction yield of S. baumii polyphenols. Moreover, the efficiency of DES to extract polyphenols were more than two times higher $(12.58 \mathrm{mg} / \mathrm{g})$ than that obtained using the conventional method with ethanol $(4.20 \mathrm{mg} / \mathrm{g})$. These findings indicated the obvious superiority of our method.

\subsection{Antioxidant Activity of S. baumii Polyphenols}

The experimental results of the antioxidant activity of polyphenols were shown in Figure 5.

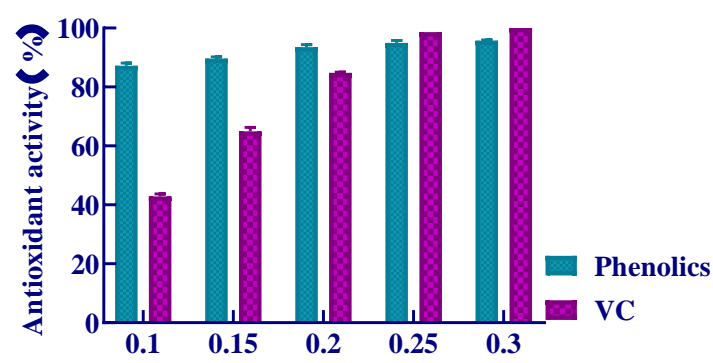

Phenolics concentration $(\mathrm{mg} / \mathrm{mL})$

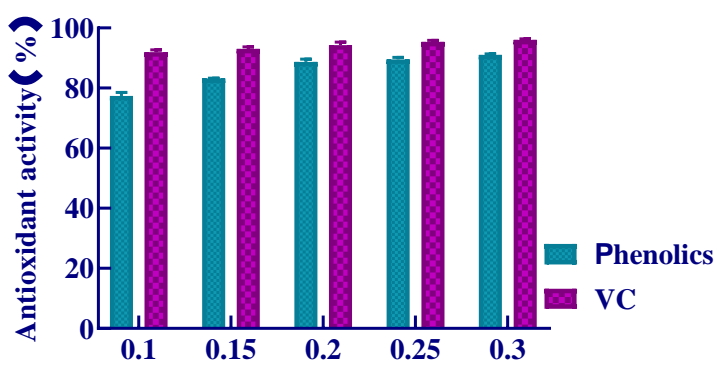

Phenolics concentration $(\mathrm{mg} / \mathrm{mL})$

(A)

(B)

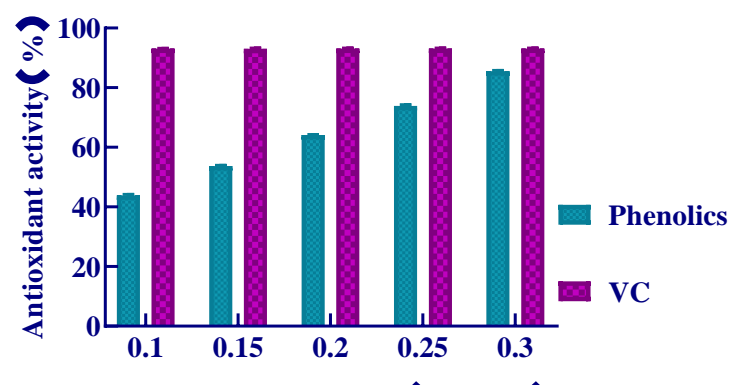

Phenolics concentration $(\mathrm{mg} / \mathrm{mL})$

(C)

Figure 5. The experimental results of antioxidant activity of polyphenols.

The $\bullet \mathrm{OH}$-scavenging activity of $S$. baumii polyphenols is higher compared with that of VC (Figure 5A). The free-radical scavenging ability of the polyphenols increased with an increase in the concentration of the extracted polyphenols. The scavenging ability was as high as $95.71 \%$ when the polyphenols concentration was $0.30 \mathrm{mg} / \mathrm{mL}$. The scavenging ability was comparable to, or even higher than, VC at low concentrations. 
The DPPH scavenging rate of S. baumii polyphenols is shown as (Figure 5B). VC was used as a control. We found an increase in the rate of DPPH scavenging with increasing polyphenols concentration. When the polyphenols concentration was $0.30 \mathrm{mg} / \mathrm{mL}$, the DPPH scavenging ability was the highest and reached $91.08 \%$, which was similar to the scavenging rate by $\mathrm{VC}$.

Results of $\mathrm{ABTS}^{+}$scavenging rate by S. baumii polyphenols are shown as (Figure 5C). $\mathrm{VC}$ was used as a control. We found that $\mathrm{ABTS}^{+}$scavenging by $S$. baumii polyphenols increased with increasing polyphenols concentration. The $\mathrm{ABTS}^{+}$-scavenging ability was the highest at $85.52 \%$ when the polyphenols concentration was $0.30 \mathrm{mg} / \mathrm{mL}$.

\section{Discussion}

Currently, studies on the active compounds of Sanghuang are mostly focused on polysaccharides, and in-depth studies on polyphenols are still limited. In this study, DES extraction was used to greatly improve the polyphenol yields of S. baumii. Although DES can better dissolve polyphenols, their solubility and extraction yields will differ based on the DES used.

For this study, we selected eight types of DESs and found that the one composed of choline chloride, and malic acid had the highest extraction yield. The polarity of the DES composed of choline chloride and malic acid is higher than that of the ethanol system. The types of polyphenols in Sanghuang are complicated. Based on our separation and analysis experiments, Sanghung is rich in some polyphenols with strong polarities, such as gallic acid, phellibaumin A, chlorogenic acid, etc. These polyphenols have strong hydrogen bond interaction with DES and can disperse into DES quickly. Therefore, The DES greatly improves the yield of polyphenols with a higher polarity in Sanghuang.

In the single factor experiments, the extraction yield of polyphenols first increased, and then decreased with an increase in water content. This was likely because when the water content was low, the viscosity of DES was high, which hindered the interaction between S. baumii and DES, thereby reducing the extraction rate. When the water content was very high, the interaction force between $S$. baumii and DES decreased, resulting in a lower extraction yield. With an increase in extraction time, the extraction rate of polyphenols increased initially but then tended to stabilize. This pattern could be explained on the basis that ultrasound treatment damaged the cell walls and promoted the dissolution of intracellular polyphenols as the time was increased. After reaching the maximum value, no further increase was observed. With an increase in temperature, the extraction rate of polyphenols increased initially and then decreased. This finding could be attributed to the increase in temperature resulting in the destruction of the cell wall, leading to the dissolution of the intracellular polyphenols. However, with this increase in temperature, some unstable polyphenolic components were destroyed, and other substances were generated, which led to a decrease in the rate of extraction. With an increase in the solid-liquid ratio, the extraction rate of polyphenols showed a trend of an initial increase, followed by the attainment of stability. This may be because the solubility property of DES did not increase after reaching saturation. In terms of pharmacological activity, our in vitro antioxidant studies suggested that the mulberry polyphenols of $S$. baumii had good antioxidant activity. Although we made some interesting discoveries, our study has some limitations. For example, the polyphenols used in the antioxidant experiments were crude compounds obtained after preliminary purification; moreover, the reusability of DES has yet to be proven. The complicated purification procedure of polyphenols is another problem that should be addressed in subsequent experiments.

In future studies, we will continue to further purify the monomer polyphenols. Additionally, we intend to perform more in vivo and in vitro experimental studies to further explore the anti-inflammatory, hypoglycemic, and antitumor effects of the polyphenols of mulberry flavors. 


\section{Materials and Methods}

\subsection{Materials, Reagents, and Instruments}

Materials: S. baumii was cultured for six months in our laboratory and used in this study. DNA kits were used for ITS sequence amplification and sent to Huada Gene Sequencing Company (Qingdao, China). The measured gene sequences were deposited in the GenBank database to Blast the sequence alignment and combined with the morphological identification, S. baumii was identified. The fruiting bodies were dried at $50-60{ }^{\circ} \mathrm{C}$, pulverized in a grinder, passed through a 40 -mesh sieve(The aperture is $0.425 \mathrm{~nm}$ ), and stored at $4{ }^{\circ} \mathrm{C}$ until further use.

Reagents: Choline chloride, lactic acid, glycerol, malonic acid, urea, glycol, 1,4butanediol, glycerol, potassium persulfate, trichloroacetic acid, ferric chloride, potassium ferricyanide, salicylic acid, sodium carbonate, ferrous sulfate, vitamin C (VC) were of analytical grade and obtained from Sinopill Chemical Reagents Co., Ltd. (Tianjin, China). Gallic acid monohydrate standard (purity $>98 \%$ ) analytical pure was purchased from Cool Chemical Technology Co., Ltd. (Beijing, China). Folin was obtained from Shanghai Jinsui Biotechnology Co., Ltd. (Shanghai, China). and analytical grade hydrogen peroxide $\left(\mathrm{H}_{2} \mathrm{O}_{2}\right)$ was procured from Tianjin Ruijin Chemical Co., Ltd. (Tianjin, China). 1,1-diphenyl2-picrylhydrazyl (DPPH), 2,2'-azino-bis(3-ethylbenzothiazoline-6-sulfonic acid) (ABTS ${ }^{+}$) was purchased from Shanghai Maclin Biochemical Technology Co., Ltd. (Shanghai, China).

Experimental apparatus: A 2500C grinder (Yongkang Hongsun Electromechanical Co., Ltd., Zhejiang, China), SQP electronic balance (Sartorius Scientific Instruments, Beijing, China), KQ-500DE ultrasonic extractor (Kunshan Ultrasonic Instrument Co., Ltd. Jiangsu, China), HHS-21-6 thermostat water bath (Shanghai Boxun Industrial Co., Ltd., Medical Equipment Factory, Shanghai, China), T6 spectrophotometer (Beijing Purkay General Instrument Co. Ltd., Beijing, China), NEO15R high-speed refrigerated centrifuge (Shanghai Lishen Scientific Instrument Co., Ltd., Shanghai, China), and DHT-450A high-temperature blast drying oven (Shanghai Daohan Industrial Co., Ltd., Shanghai, China) were the instruments used in this study.

\subsection{Method}

\subsubsection{Preparation of DES}

DES is composed of two parts: the hydrogen donor (choline chloride) and hydrogen acceptor (malic acid, lactic acid, etc.). The two parts were weighed in a certain molar ratio, placed in a round-bottomed flask, and heated in a water bath at $80-90^{\circ} \mathrm{C}$ for $4-6 \mathrm{~h}$ with constant stirring until a colorless transparent liquid was obtained. The hydrogen bonding force between the compounds causes the high viscosity of DES. The addition of a certain volume of water can reduce viscosity and enable the sample to effectively combine with the eutectic solvent. In this study, different eutectic solvents were synthesized as listed in Table 3.

Table 3. Composition of DES.

\begin{tabular}{ccc}
\hline Number & Composition & Molar Ratio \\
\hline DES-1 & Choline chloride: Malic acid & $1: 1$ \\
DES-2 & Choline chloride: Lactic acid & $1: 1$ \\
DES-3 & Choline chloride: Glycerol & $1: 2$ \\
DES-4 & Choline chloride: Malonic acid & $1: 1$ \\
DES-5 & Choline chloride: Urea & $1: 2$ \\
DES-6 & Choline chloride: Oxalic acid & $1: 1$ \\
DES-7 & Choline chloride: 1,4-Butanediol & $1: 5$ \\
DES-8 & Choline chloride: Urea: Glycerol & $1: 1: 1$ \\
\hline
\end{tabular}

\subsubsection{Construction of the Standard Curve}

Polyphenol content was determined using Folinol colorimetry [30] with some modifications. Gallic acid monohydrate was used as a standard; $0.1000 \mathrm{~g}$ of gallic acid was 
accurately weighed, dissolved in distilled water, and made up to a volume of $100 \mathrm{~mL}$ in a volumetric flask to obtain a $1 \mathrm{mg} / \mathrm{mL}$ standard solution. The standard solution $(0,1,2,3,4$, and $5 \mathrm{~mL}$ ) was each pipetted into a $100-\mathrm{mL}$ volumetric flask and the volume was made up with distilled water. The mass concentrations of the prepared solutions were $0,10,20$, 30,40 , and $50 \mathrm{mg} / \mathrm{L}$, respectively. The sample $(1 \mathrm{~mL})$ was taken in a $10-\mathrm{mL}$ volumetric flask and $1 \mathrm{~mL}$ of Folinol reagent was added. The mixture was allowed to stand for $5 \mathrm{~min}$, following which $3 \mathrm{~mL}$ of $7.5 \% \mathrm{Na}_{2} \mathrm{CO}_{3}$ was added and the volume was made up to $10 \mathrm{~mL}$ with distilled water. Three replicates were performed for each dilution. The samples were mixed well and left undisturbed in the dark for $2 \mathrm{~h}$. Lastly, the absorbance was determined at $765 \mathrm{~nm}$ using appropriate blanks. The mass concentration of gallic acid was plotted as the abaxial coordinate and the absorbance as the vertical coordinate to obtain the standard curve. The regression equation was determined to be $y=0.0127 x+0.0001\left(R^{2}=0.9998\right)$.

\subsubsection{Determination of Polyphenols Yield}

The fruiting body powder of $S$. baumii was weighed and a proportional volume of DES was added. The sample was subjected to ultrasonic-assisted extraction in accordance with the different extraction conditions. Next, the sonicated samples were centrifuged at $5000 \mathrm{rpm}$ for $10 \mathrm{~min}$. The supernatant obtained after centrifugation was diluted according to the method stated in Section 4.2.2 and the polyphenol concentration was determined. Polyphenols content was calculated using the following Equation (1):

$$
\text { Polyphenol content }(m g / g))=(C * V * n) / m
$$

where $\mathrm{C}$ is the mass concentration of the extract $(\mathrm{mg} / \mathrm{mL}), \mathrm{V}$ is the volume of the solution $(\mathrm{mL}), \mathrm{n}$ is the dilution factor, and $\mathrm{m}$ is the dry weight of the sample $(\mathrm{g})$.

\subsection{Single-Factor Experiment of Ultrasonic-Assisted Extraction of Polyphenols from S. baumii Using DES}

4.3.1. Effect of Water Content on the Extraction Yield of Polyphenols from S. baumii Using DES

The fruiting-body powder of $S$. baumii was dissolved to yield a $50 \mathrm{mg} / \mathrm{mL}$ solution. DES with a water content of $20 \%, 30 \%, 40 \%, 50 \%$, and $60 \%$ were used to extract the samples in an ultrasonicator operated with an extraction power of $200 \mathrm{~W}, 40 \mathrm{kHz}$, extraction temperature of $50{ }^{\circ} \mathrm{C}$, and extraction time of $30 \mathrm{~min}, 5 \mathrm{~mL}$ were extracted respectively. Each experiment was performed in triplicate. The optimal water content of DES was determined by comparing the amount of phenolic content obtained from each extraction.

\subsubsection{Effect of Extraction Time on Polyphenol Yield from S. baumii}

The fruiting-body powder of $S$. baumii was dissolved to yield a $50 \mathrm{mg} / \mathrm{mL}$ solution. DES with $40 \%$ water content were used to extract the samples in the ultrasonic extractor with an extraction power of $200 \mathrm{~W}, 40 \mathrm{kHz}$, extraction temperature of $50{ }^{\circ} \mathrm{C}$, and extraction time of 20, 30, 40, 50, and $60 \mathrm{~min}, 5 \mathrm{~mL}$ were extracted respectively. Each experiment was performed in triplicate. The optimal extraction time was determined by comparing the number of polyphenols extracted at the end of each time point.

\subsubsection{Effect of Extraction Temperature on the Extraction Yield of Polyphenols from} S. baumii

The fruiting-body powder of $S$. baumii was dissolved to yield a $50 \mathrm{mg} / \mathrm{mL}$ solution. DES with $40 \%$ water content were used to extract the samples in an ultrasonic extractor with an extraction power of $200 \mathrm{~W}, 40 \mathrm{kHz}$, extraction time of $30 \mathrm{~min}$ and extraction temperature of $40^{\circ} \mathrm{C}, 50{ }^{\circ} \mathrm{C}, 60^{\circ} \mathrm{C}, 70^{\circ} \mathrm{C}$, and $80^{\circ} \mathrm{C}, 5 \mathrm{~mL}$ were extracted respectively. Each experiment was performed in triplicate. The optimal extraction temperature was determined by comparing the number of polyphenols from each extraction. 


\subsubsection{Effect of Solid-Liquid Ratio on the Extraction Yield of Polyphenols from S. baumii}

The fruiting body powder of $S$. baumii was dissolved to yield concentrations of 30, 40, 50,60 , and $70 \mathrm{mg} / \mathrm{mL}$. DES with $40 \%$ water content were used to extract samples in an ultrasonic extractor with an extraction power of $200 \mathrm{~W}, 40 \mathrm{kHz}$, extraction temperature of $50{ }^{\circ} \mathrm{C}$, and extraction time of $30 \mathrm{~min}, 5 \mathrm{~mL}$ were extracted respectively. To ensure accuracy, each experiment was performed in triplicate. The optimal solid-liquid ratio was determined by comparing the number of polyphenols obtained from each extraction.

\subsection{Optimization of the Extraction Process of Polyphenols Using Response Surface Methodology}

According to the results of the single-factor experiment and the experimental principle of Box-Behnken in the response surface curve method, response surface analysis was performed with four factors and three levels using Desk-Expert 12 software (Table 4). The water content of the DES, extraction time, extraction temperature, and solid-liquid ratio were selected as the main influencing factors. Three levels, namely, low, medium, and high were designed for each factor and marked $-1,0$, and 1 , respectively.

Table 4. Factors and levels in response surface analysis.

\begin{tabular}{cccc}
\hline Factors & \multicolumn{2}{c}{ Level } \\
\cline { 2 - 4 } & $\mathbf{- 1}$ & $\mathbf{0}$ & $\mathbf{1}$ \\
\hline A ratio of the material liquid $(\mathrm{mg} / \mathrm{mL})$ & 30 & 40 & 50 \\
B Extraction time $(\mathrm{min})$ & 30 & 40 & 50 \\
C Extraction temperature $\left({ }^{\circ} \mathrm{C}\right)$ & 50 & 60 & 70 \\
D The water content $(\%)$ & 30 & 40 & 50 \\
\hline
\end{tabular}

\subsection{In Vitro Antioxidant Activity of S. baumii Polyphenols}

The deep eutectic solvent extract was purified by macroporous resin(AB-8) to obtain crude polyphenols. Then the obtained crude polyphenols were prepared into sample solution with concentrations of $0.10,0.15,0.20,0.25$, and $0.30 \mathrm{mg} / \mathrm{mL}$, respectively, for reserve.

\subsection{1. •OH-Scavenging Activity of S. baumii Polyphenols}

The scavenging ability of polyphenols on $\bullet \mathrm{OH}$ was determined using the $\mathrm{H}_{2} \mathrm{O}_{2} / \mathrm{Fe}$ method, following the method reported by Smirnoff [31] with slight modifications. VC was used as a control, and $9 \mathrm{mmol} / \mathrm{L} \mathrm{FeSO}_{4}$ and $9 \mathrm{mmol} / \mathrm{L}$ salicylic acid-ethanol were prepared for later use. A volume of $250 \mu \mathrm{L}$ of $30 \% \mathrm{H}_{2} \mathrm{O}_{2}$ was diluted to $250 \mathrm{~mL}$ with water and stored away from light in an amber reagent bottle until used.

In a test tube, $1 \mathrm{~mL} \mathrm{FeSO}_{4}, 2 \mathrm{~mL}$ salicylic acid-ethanol solution, and $2 \mathrm{~mL}$ distilled water were mixed, and $2 \mathrm{~mL} \mathrm{H}_{2} \mathrm{O}_{2}$ solution was added and quickly mixed. The sample was incubated for $1 \mathrm{~h}$ in a water bath at $37^{\circ} \mathrm{C}$, and the absorbance was measured at $510 \mathrm{~nm}$ to obtain the value for the blank control group, $\mathrm{A}_{0}$.

Next, $1 \mathrm{~mL} \mathrm{FeSO}_{4}, 2 \mathrm{~mL}$ salicylic acid-ethanol solution, and $2 \mathrm{~mL}$ of distilled water, were added to $1 \mathrm{~mL}$ of the extract obtained using different concentration gradients $(0.10$, $0.15,0.20,0.25$, and $0.30 \mathrm{mg} / \mathrm{mL}$ ). After mixing, the samples were incubated in a water bath at $37^{\circ} \mathrm{C}$ for $1 \mathrm{~h}$. The absorbance was measured at $510 \mathrm{~nm}$ and readings for the blank reagent group were obtained, which were labeled as $\mathrm{A}_{\mathrm{y}}$.

Lastly, $1 \mathrm{~mL}$ of the extract obtained using different concentration gradients $(0.10,0.15$, $0.20,0.25$, and $0.30 \mathrm{mg} / \mathrm{mL}$ ) was added to $1 \mathrm{~mL} \mathrm{FeSO}_{4}$ and $2 \mathrm{~mL}$ salicylic acid-ethanol solution in the respective test tubes. After mixing, $2 \mathrm{~mL}$ of $\mathrm{H}_{2} \mathrm{O}_{2}$ was added. After rapid mixing, the samples were incubated in a water bath at $37^{\circ} \mathrm{C}$ for $1 \mathrm{~h}$, and the absorbance was determined at $510 \mathrm{~nm}$ to obtain readings for the sample group, marked as $\mathrm{A}_{\mathrm{x}}$. The Scavenging rate was calculated using the following Equation (2):

$$
\text { Scavenging rate }(\%)=\frac{\mathrm{A}_{0}-\left(\mathrm{A}_{\mathrm{x}}-\mathrm{A}_{\mathrm{y}}\right)}{\mathrm{A}_{0}} \times 100 \%
$$




\subsubsection{DPPH-Scavenging Activity of S. baumii Polyphenols}

The DPPH-scavenging ability of polyphenols was determined using the method reported by Xican Li [32] with slight modifications. VC was used as the control. A $0.004 \%$ DPPH-ethanol solution was prepared and stored in a refrigerator at $4{ }^{\circ} \mathrm{C}$ until used. Sample solutions of different concentrations $(0.10,0.15,0.20,0.25$, and $0.30 \mathrm{mg} / \mathrm{mL})$ were added into the respective test tubes, followed by the addition of $1 \mathrm{~mL}$ of $0.0004 \% \mathrm{DPPH}$ solution and $2 \mathrm{~mL}$ of anhydrous ethanol. After thorough mixing, the reaction was allowed to progress by keeping the test tubes undisturbed in the dark for $30 \mathrm{~min}$. The absorbance was measured at $517 \mathrm{~nm}$ and marked as $A_{x}$. Distilled water was used as a blank and marked as $\mathrm{A}_{0}$. The blank control group was set as a sample, which was marked as $\mathrm{A}_{\mathrm{y}}$. The Scavenging rate was calculated using the following Equation (3):

$$
\text { Scavenging rate }(\%)=\frac{A_{0}-\left(A_{x}-A_{y}\right)}{A_{0}} \times 100 \%
$$

\subsubsection{ABTS ${ }^{+}$-Scavenging Activity of S. baumii Polyphenols}

The ABTS ${ }^{+}$-scavenging ability of polyphenols was determined using the method reported by Miller [33] with slight modifications. VC was used as a control. ABTS $(7.4 \mathrm{mmol} / \mathrm{L})$ was mixed with $2.6 \mathrm{mmol} / \mathrm{L} \mathrm{K}_{2} \mathrm{~S}_{2} \mathrm{O}_{8}$ in a $1: 1$ ratio, and the reaction was allowed to progress in the dark at $25^{\circ} \mathrm{C}$ for $12 \mathrm{~h}$. After a 40-50-fold dilution, the absorbance was measured at $734 \mathrm{~nm}$. Sample solutions of different concentrations $(0.10,0.15,0.20,0.25$, and $0.30 \mathrm{mg} / \mathrm{mL}$ ) were added to $4 \mathrm{~mL}$ of $\mathrm{ABTS}^{+}$working solution, mixed thoroughly, and allowed to stand for $6 \mathrm{~min}$. The absorbance was determined at $734 \mathrm{~nm}$ and marked as A. Anhydrous ethanol was used as a blank control and marked as $\mathrm{A}_{0}$. The Scavenging rate was calculated using the following Equation (4):

$$
\text { Scavenging rate }(\%)=\frac{A_{0}-\mathrm{A}}{A_{0}} \times 100 \%
$$

\section{Conclusions}

In this study, we have reported a novel method for the extraction of polyphenols from S. baumii using DES. In the single-factor experiment, using the same extraction conditions (solid-liquid ratio of $50 \mathrm{mg} / \mathrm{mL}$, ultrasonic-assisted extraction for $30 \mathrm{~min}$, temperature of $50{ }^{\circ} \mathrm{C}$, power of $200 \mathrm{~W}, 40 \mathrm{kHz}$ ), we compared the results obtained using ethanol extraction (60\% ethanol was found to be the optimal extraction condition). When DES was used for extraction, the polyphenol yield was $6.37 \mathrm{mg} / \mathrm{g}(\mathrm{DES}-1)$, which was 1.5 times more than that obtained using $60 \%$ ethanol $(4.20 \mathrm{mg} / \mathrm{g})$. These findings preliminarily indicate the extraction yield of polyphenols using a eutectic solvent. Based on our initial findings, we used response surface analysis to further enhance the extraction conditions using DES. The ideal extraction time was $42 \mathrm{~min}$, the extraction temperature was $58{ }^{\circ} \mathrm{C}$, the solidliquid ratio was $34 \mathrm{mg} / \mathrm{mL}$, and the water content was $39 \%$. Using these conditions, the polyphenol yield from $S$. baumii was found to be $12.58 \mathrm{mg} / \mathrm{g}$, which was about 2 times as much as before conditional optimization $(6.37 \mathrm{mg} / \mathrm{g})$. This result demonstrated the superiority of DES to extract polyphenols. When the concentration of the polyphenols was $0.30 \mathrm{mg} / \mathrm{mL}$, the $\bullet \mathrm{OH}-$, DPPH-, and $\mathrm{ABTS}^{+}$- scavenging rates were $95.71 \%, 91.08 \%$, and $85.52 \%$, respectively. These findings indicated that $S$. baumii polyphenols were potent-free radical scavengers and natural antioxidants, further highlighting their broad application prospects in drug development and the research and development of health supplements.

Author Contributions: R.Z. and X.C. designed this study and led the project. N.Z., Y.M. and J.C. performed the experiments, analyzed the data, and wrote the manuscript. S.Y., G.W. and W.L. edited and revised the manuscript. All authors have read and agreed to the published version of the manuscript. 
Funding: This work was supported by the Yantai Science and Technology Development Project, Shandong Province, China (2019XDHZ092), the Shandong Province Science and Technology Development Project, China (2019JZZY010717), Edible Fungus Industrial System Post Expert of Modern Agricultural Industrial Technology System of Shandong Province, Genetic Breeding Post, Lunong Technology (2016) No. 18, and the Start-up funding from Ludong University(ZR2021013), Shandong Province, China.

Institutional Review Board Statement: Not applicable.

Informed Consent Statement: Not applicable.

Data Availability Statement: All the data is shown in the article, andthe study did not report any data.

Conflicts of Interest: The authors declare no conflict of interest.

Sample Availability: Samples of the compounds of S. baumii are available from the authors.

\section{References}

1. Chengshan, C.; Jiexin, M.; Chunrui, H.; Yi, J.; Guozhu, Z.; Xiangwei, H. Extraction and antioxidant activity of total triterpenoids in the mycelium of a medicinal fungus, Sanghuangporus sanghuang. Sci. Rep. 2019, 9, 1-10.

2. Zhang, W.-B.; Wang, J.-G.; Li, Z.-K.; Yang, L.-Q.; Qin, J.; Xiang, Z.-H.; Ciu, H.J. Research Progress on Medicinal Fungus Sanghuang. China J. Chin. Mater. Med. 2014, 39, 2838-2845.

3. Wu, S.-H. Name correction of precious medicinal fungus "sanghuang" species. Edible Med. Mushrooms 2012, 20, 177-179.

4. Wu, S.-H.; Dai, Y.-C. Species clarification of the medicinal fungus Sanghuang. Mycosystema 2020, 39, 781-794.

5. Bao, H.-Y.; Yang, S.; Li, Q.-J.; Bau, T.; Li, Y. Supplementary Textual Research on "Sanghuang”. J. Fungal Res. 2017, 15, 264-270.

6. Cheng, J.; Song, J.; Wei, H.; Wang, Y.; Huang, X.; Liu, Y.; Lu, N.; He, L.; Lv, G.; Ding, H.; et al. Structural characterization and hypoglycemic activity of an intracellular polysaccharide from Sanghuangporus sanghuang mycelia. Int. J. Biol. Macromol. 2020, 164, 3305-3314. [CrossRef]

7. Song, T.; Zhang, Z.; Jin, Q.; Feng, W.; Shen, Y.; Fan, L.; Cai, W. Nutrient profiles, functional compositions, and antioxidant activities of seven types of grain fermented with Sanghuangporus sanghuang fungus. J. Food Sci. Technol. 2020, 1-11. [CrossRef]

8. Zhu, Z.-P.; Li, N. Antioxidant Properties of Polysaccharides from Phellinus igniarius in vitro. Food Sci. 2011, 32, 92-95.

9. Ye, Y.-J.; Shi, G.; Zhou, Z.-Y.; Wang, J.-Q.; An, L.-P.; Du, P.-G. Comparative Chemical Composition of Mycelium and Fruit Body of Phellinus igniarius. Food Sci. 2019, 40, 246-251.

10. Liu, K.; Xiao, X.; Wang, J.; Chen Oliver, C.-Y.; Hu, H. Polyphenolic composition and antioxidant, antiproliferative, and antimicrobial activities of mushroom Inonotus sanghuang. LWT Food Sci. Technol. 2017, 82, 154-161. [CrossRef]

11. Zan, L.-F.; Bao, H.-Y.; Li, D.-H. Review on Polyphenol Components from Medicinal Fungi "Sanghuang" and Their Biological Activity. Nat. Prod. Res. Dev. 2016, 28, 147-155.

12. Hwang, B.S.; Lee, I.-K.; Choi, H.J.; Yun, B.-S. Anti-influenza activities of polyphenols from the medicinal mushroom Phellinus baumii. Bioorg. Med. Chem. Lett. 2015, 25, 3256-3260. [CrossRef] [PubMed]

13. Suabjakyong, P.; Saiki, R.; Van Griensven, L.J.L.D.; Higashi, K.; Nishimura, K.; Igarashi, K.; Toida, T. Polyphenol Extract from Phellinus igniarius Protects against Acrolein Toxicity In Vitro and Provides Protection in a Mouse Stroke Model. PLoS ONE 2015, 10, e122733. [CrossRef] [PubMed]

14. Wu, C.-S.; Lin, Z.-M.; Wang, L.-N.; Guo, D.-X.; Wang, S.-Q.; Liu, Y.-Q.; Yuan, H.-Q.; Lou, H.-X. Phenolic compounds with NF-kB inhibitory effects from the fungus Phellinus baumii. Bioorg. Med. Chem. Lett. 2011, 21, 3261-3267. [CrossRef]

15. Lin, W.-C.; Deng, J.-S.; Huang, S.-S.; Wu, S.-H.; Chen, C.-C.; Lin, W.-R.; Lin, H.-Y.; Huang, G.-J. Anti-Inflammatory Activity of Sanghuangporus sanghuang Mycelium. Int. J. Mol. Sci. 2017, 18, 347. [CrossRef]

16. $\mathrm{Xu}, \mathrm{X}$.; Li, J.; Hu, Y. Polysaccharides from Inonotus obliquus sclerotia and cultured mycelia stimulate cytokine production of human peripheral blood mononuclear cells in vitro and their chemical characterization. Int. Immunopharmacol. 2014, 21, 269-278. [CrossRef]

17. Friedman, M. Mushroom Polysaccharides: Chemistry and Antiobesity, Antidiabetes, Anticancer, and Antibiotic Properties in Cells, Rodents, and Humans. Foods 2016, 5, 80. [CrossRef]

18. Jung, G.H.; Kang, J.H. Efficacy of Phellinus linteus (sanghuang) extract for improving immune functions. Medicine 2020, 99, e18829. [CrossRef]

19. Mané, C.; Souquet, J.M.; Ollé, D.; Verriés, C.; Véran, F.; Mazerolles, G.; Cheynier, V.; Fulcrand, H. Optimization of simultaneous flavanol, phenolic acid, and anthocyanin extraction from grapes using an experimental design: Application to the characterization of champagne grape varieties. J. Agric. Food Chem. 2007, 55, 7224-7233. [CrossRef]

20. Cheng, C.; Hu, W.-Z.; Tian, P.-Y. Optimization of Ultrasound-Assisted Extraction of Polyphenols from Banana Peel and Their Antioxidant Capacity. Food Sci. 2014, 35, 12-17. [CrossRef] 
21. Hayat, Z.; Hussain, S.; Abbas, S.; Umar, F.; Ding, B.; Xia, S.; Jia, C.; Zhang, X.; Xia, W. Optimized microwave-assisted extraction of phenolic acids from citrus mandarin peels and evaluation of antioxidant activity in vitro. Sep. Purif. Technol. 2009, 70, 63-70. [CrossRef]

22. Zheng, H.-Z.; Hwang, I.-W.; Kim, S.-K.; Lee, S.-H.; Chung, S.-K. Optimization of carbohydrate-hydrolyzing enzyme aided polyphenol extraction from unripe apples. J. Korean Soc. Appl. Biol. Chem. 2010, 53, 342-350. [CrossRef]

23. Carla, D.P.; Andrea, N. Supercritical fluid extraction of polyphenols from grape seed (Vitis vinifera): Study on process variables and kinetics. J. Supercrit. Fluids 2017, 130, 239-245.

24. Luo, X.; Cui, J.; Zhang, H.; Duan, Y. Subcritical water extraction of polyphenolic compounds from sorghum (Sorghum bicolor L.) bran and their biological activities. Food Chem. 2018, 262, 14-20. [CrossRef]

25. Abbott, A.P.; Capper, G.; Davies, D.L.; Rasheed, R.K.; Tambyrajah, V. Novel solvent properties of choline chloride/urea mixtures. Chem. Commun. 2003, 70-71. [CrossRef] [PubMed]

26. Wei, Z.-F.; Wang, X.-Q.; Peng, X.; Wang, W. Fast and green extraction and separation of main bioactive flavonoids from Radix Scutellariae. Ind. Crops Prod. 2015, 63, 175-181. [CrossRef]

27. Zhang, Q.; De Oliveira Vigier, K.; Royer, S.; Jérôme, F. Deep eutectic solvents: Syntheses, properties and applications. Chem. Soc. Rev. 2012, 41, 7108-7146. [CrossRef]

28. Wentao, B.; Minglei, T.; Kyung, H.R. Evaluation of alcohol-based deep eutectic solvent in extraction and determination of flavonoids with response surface methodology optimization. J. Chromatogr. A 2013, 1285, 22-30.

29. Peng, X.; Duan, M.-H.; Yao, X.-H.; Zhao, C.-J.; Zu, Y.-G.; Fu, Y.-J. Green extraction of five target phenolic acids from Lonicerae japonicae Flos with deep eutectic solvent. Sep. Purif. Technol. 2016, 157, 249-257. [CrossRef]

30. Li, J.-X.; Zhang, X.-N.; Li, W.-W. Comparative Studies of Total Anthocyanins, Total Polyphenols and Antioxidant Activities of Different Pomegranate Varieties. Food Sci. 2011, 32, 143-146.

31. Smirnoff, N.; Cumbes, Q.J. Hydroxyl radical scavenging activity of compatible solutes. Pergamon 1989, 28, 1057-1060. [CrossRef]

32. Li, X. Comparative Study of 1,1-Diphenyl-2-picryl-hydrazyl Radical (DPPH•) Scavenging Capacity of the Antioxidant Xanthones Family. Chem. Sel. 2018, 3, 13081-13086. [CrossRef]

33. Miller, N.J.; Rice-Evans, C.; Davies, M.J.; Gopinathan, V.; Milner, A. A novel method for measuring antioxidant capacity and its application to monitoring the antioxidant status in premature neonates. Clin. Sci. 1993, 84, 407-412. [CrossRef] [PubMed] 